\title{
Region specific differences in the effect of propofol on the murine colon result in dysmotility
}

Lucy B Diss ${ }^{1}$, Shannon Villeneuve ${ }^{2}$, Kim R Pearce ${ }^{1}$, Mark S. Yeoman ${ }^{2,3}$, and Bhavik A. Patel ${ }^{\star 2,3}$

${ }^{1}$ Brighton and Sussex Medical School, Brighton, UK; ${ }^{2}$ School of Pharmacy and Biomolecular Sciences and ${ }^{3}$ Centre for Stress and Age-Related Diseases, University of Brighton, Brighton, UK

*Corresponding Author: Dr Bhavik A. Patel, b.a.patel@brighton.ac.uk, tel: $+44(0) 1273642418$

Running title: Propofol cause colonic dysmotility 


\begin{abstract}
Propofol is the most widely used intravenous anaesthetic agent for maintenance of anaesthesia and sedation. Studies in varying regions of the bowel have shown conflicting differences on the effects of propofol on motility. There the aim of this study was to understand the influence of propofol on colonic function and explore by which mechanism any changes occur. Functional studies were conducted using isolated colonic tissue from C57BL6 mice which were exposed to $5 \mu \mathrm{M}$ propofol. Faecal pellet motility, colonic migratory motor complexes (CMMCs) and functional bioassays were utilised to monitor colonic function and nitric oxide production was monitored by amperometry. There was a signficant reduction in amplitude of CMMCs in the distal colon in the presence of $5 \mu \mathrm{M}$ propofol, however no difference was observed in the proximal colon. A signficant increase in the 5-HT evoked contractions were observed in distal colon in the presence of $5 \mu \mathrm{M}$ propofol. Additionally, a reduction in the NO production in the presence of $5 \mu \mathrm{M}$ propofol was only observed in the in the distal colon. As a result, in the presence of $5 \mu \mathrm{M}$ propofol, faecal pellet transit was increased, and velocity was reduced. At clinically relevant doses, propofol was shown to reduce colonic motility by inhibiting nitric oxide synthase in only the distal region of the colon. Our findings indicate that propofol has a considerable influence on colonic signalling mechanisms and impairs colonic motility, which may have implications in its clinical use especially for maintenance.
\end{abstract}

Keywords: Propofol, proximal colon, distal colon, nitric oxide, colonic transit, colonic migratory motor complexes

\title{
1. Introduction
}

Propofol (2,6-diisopropylphenol) is the most widely used intravenous anaesthetic agent for induction and maintenance of anaesthesia and sedation. Its short elimination 
half-life enables a faster more efficient recovery of the patient post-operatively compared to more volatile agents such as sodium thiopentone(Favetta et al., 2002). In intensive care units (ICU) it is a successful sedative in controlling delirium in critically ill patients(Rowe et al., 2008). Propofol circulates the body for longer periods of time when used as a maintenance anaesthetic for sedation and some studies have explored the complications associated with its use. However little focus was placed on gastrointestinal function(Beller et al., 1988; Venn et al., 2001). Therefore, there is uncertainty of the side effects of prolonged propofol use on gastrointestinal function.

Very few studies have been conducted into the effects of propofol on the gastrointestinal tract. Studies have shown that propofol decreases motility in the duodenum(Russell et al., 1989), reduces gastric emptying and increases gastrointestinal transit(Inada et al., 2004). Propofol has also been shown to decrease ACh-dependent contractility in human gastric and colonic smooth muscle at concentrations within the therapeutic range for sedation and total intravenous anaesthesia (TIVA)(Lee et al., 1999). However, other studies have shown that propofol, even when given as a continuous infusion, does not alter gastrointestinal tract motility more than the standard isoflurane anaesthesia(Freye et al., 1998). Another investigation have explored the effect of propofol-fentanyl infusions on gastrointestinal function. They demonstrated impaired duodenal motility, however whether this change was due to the propofol or the opioid was unclear(Schnoor et al., 2005).

With all these studies little is known on the mechanism underlying changes on gastrointestinal motility. Therefore, there is confusion on the precise effect of propofol in the gastrointestinal tract and little is known about the effects in specific regions of the bowel. There is also a limited understanding of the mechanisms by which propofol alters gastrointestinal transit. The effects of propofol on the colon are not well known and therefore this study aims to explore the effects of a maintenance dose of propofol on specific regions of the colon. 


\section{Methods}

\subsection{Animals and tissue preparation}

All procedures were carried out according to U.K. Home Office regulations and the Animal [Scientific Procedures] Act 1986 and were approved by the University of Brighton Ethics Committee. Animal studies were also reported in compliance with ARRIVE guidelines. Wildtype C57BL/6J male mice (6-8 weeks, Harlan UK) were group housed in cages with free access to food and tap water and maintained on a $12 \mathrm{~h}$ lightdark cycle in a temperature and humidity-controlled room. Animals were asphyxiated with $\mathrm{CO}_{2}$ and exsanguinated following cervical dislocation. The whole colon was harvested $1 \mathrm{~cm}$ proximal to the anus and placed in oxygenated $\left(95 \% \mathrm{O}_{2}\right.$ and $\left.5 \% \mathrm{CO}_{2}\right)$ Krebs' buffer solution, pH $7.4(117 \mathrm{mM} \mathrm{NaCl}, 4.7 \mathrm{mM} \mathrm{KCl}, 2.5 \mathrm{mM} \mathrm{CaCl}, 1.2 \mathrm{mM}$ $\mathrm{MgCl}_{2}, 1.2 \mathrm{mM} \mathrm{NaH}_{2} \mathrm{PO}_{4}, 25 \mathrm{mM} \mathrm{NaHCO}_{3}$ and $11 \mathrm{mM}$ glucose).

\subsection{Measurement of colonic migratory motor complexes (CMMCs)}

Briefly the whole colon was placed in a Sylgard-lined recording chamber and a thin metal rod ( $1 \mathrm{~mm}$ diameter) placed through the lumen and secured at each end to the Sylgard. The colon was continuously perfused with Krebs buffer at $5 \mathrm{ml} / \mathrm{min}$. Recordings of circular muscle contractions were made at two locations along the whole isolated colon, one at the proximal end and one at the distal end. Fine suture silk was tied through the muscle layers at each location and connected to two separate isometric force transducers. The muscle was placed initially under a low level of tension $0.4 \mathrm{~g}$ and then tension increased over the next 40 minutes until a final tension of $0.6 \mathrm{~g}$ was reached. The signal from each force transducer then passed to a preamplifier and ADI Powerlab (ADI Instruments, Oxford, UK) before being stored on computer using LabChart 7 software (ADI Instruments, Oxford, UK). The tissue was left for 30 minutes prior to recording. Post this period, recordings of spontaneous CMMCs were made for 60 minutes before the sequential addition of $5 \mu \mathrm{M}$ propofol and $5 \mu \mathrm{M}$ propofol $+100 \mu \mathrm{M}$ L-NNA (all prepared in Krebs buffer). The bath was equilibrated for 30 minutes with each pharmacological addition before spontaneous CMMCs were again recorded for 60 minutes. 


\subsection{Functional bioassay studies}

Segments of proximal and distal colon obtained from the same animal were suspended vertically in a $10 \mathrm{~mL}$ organ bath containing Krebs buffer solution at $37^{\circ} \mathrm{C}$. Tissues were initially put under $0.2 \mathrm{~g}$ tension and allowed to equilibrate for 30 minutes, in which the Krebs buffer solution was changed every 15 minutes. Concentration response curves were obtained using serotonin $(5-\mathrm{HT})$ in the concentration range of $30 \mu \mathrm{M}$ to $100 \mathrm{nM}$. Tissues were exposed to $5-\mathrm{HT}$ for one minute within the 10 dosing cycle. These concentration response curves were repeated when tissues were incubated sequentially for 30 minutes with $5 \mu \mathrm{M}$ propofol in the presence and absence of $100 \mu \mathrm{M}$ L-NNA).

\subsection{Measurement of NO production}

Longitudinal muscle myenteric plexus (LMMP) preparations were perfused with warm $\left(37^{\circ} \mathrm{C}\right)$ Krebs buffer solution at a flow rate of $2 \mathrm{~mL} \mathrm{~min}^{-1}$. Amperometric measurements were made using a $40 \mu \mathrm{m}$ boron-doped diamond (BDD) microelectrode as previously described(Patel, 2011) and a stainless steel wire served as the counter electrode and a "no leak" Ag|AgCl electrode (EE009, ESA Biosciences Inc., Sunnyvale, CA) was used as the reference electrode. Amperometric measurements were carried out using a BioStat ${ }^{\mathrm{TM}}$ multimode potentiostat (ESA Biosciences). The BDD microelectrode was placed on a myenteric ganglion using a micromanipulator (Model 25033, Fine Scientific Tools, North Vancouver, BC). The electrode was held at a detection potential of $+1.0 \mathrm{~V}$ vs. $\mathrm{Ag} \mid \mathrm{AgCl}$, which was sufficient to oxidize $\mathrm{NO}$ at a mass-transfer limited rate(MacEachern et al., 2015; Patel et al., 2008). To evoke NO release from the myenteric plexus, a local superfusion pipette was placed within $100 \mu \mathrm{m}$ of the tissue, and the tissue superfused with $10 \mu \mathrm{M}$ veratridine for $10 \mathrm{~s}$ (Sigma-Aldrich, UK). 


\subsection{Faecal pellet motility studies}

The whole colon was harvested and placed in ice cold Krebs buffer solution. The mesentery was trimmed using fine scissors and the whole colon was then loosely pinned in a Sylgard-lined flow bath, allowing a lateral movement of approximately 0.5 $\mathrm{cm}$ about the mid-line and perfused with oxygenated Krebs buffer solution at $37 \pm 1$ ${ }^{\circ} \mathrm{C}$ at a flow rate of $8 \mathrm{ml} \mathrm{min}^{-1}$. A small $(2 \mathrm{~mm})$ incision was made in both ends of the colon and the openings pinned flat to facilitate pellet insertion and its expulsion at the distal end. If spontaneous evacuation was not achieved, the faecal pellets were removed from the isolated colon after 30 minutes, by gently flushing the lumen of the colon with warmed Krebs buffer solution. The colon was then left to stabilise for 15 minutes, prior to recordings of pellet motility.

Measurements of motility were carried out using an epoxy-coated natural faecal pellet to prevent disintegration. The coated faecal pellet was inserted 3-4 $\mathrm{mm}$ into the proximal end of the bowel using a fire-polished glass capillary and the movement of the pellet was monitored using a video camera. Pellet motility was tracked using EthoVision XT video tracking software (Noldus). Following a successful trial, the experiment was repeated two further times and the average response was utilised in the presence and absence of $5 \mu \mathrm{M}$ propofol.

\subsection{Statistical Analysis}

CMMC amplitude was measured from baseline to the peak of the contraction and the duration was obtained from start to end of the contraction at baseline. The velocity of CMMCs was determined by dividing the distance between the two transducers by the time difference between the peak of proximal contraction to the peak of the closest distal contraction. For functional bioassay measurements, data were recorded using ADI Instruments bridge amplifier and Labchart 7 software. Responses were plotted as the integral of the 5-HT stimulated response minus the integral of an equivalent time period immediately preceding the stimulus. All data were normalised to the wet weight of the tissue, so data are presented as g.s mg-1. For the determination of NO release, the current responses obtained were converted to equivalent concentrations of $\mathrm{NO}$ using a calibration curve constructed using pure NO gas(Patel et al., 2006). For colonic 
motility studies, the distance travelled over time was obtained from Ethovision tracking software (Ethovision XT vs7) and the resultant velocity was obtained in the presence and absence of propofol.

Data on the CMMCs were statistically analysed using a one-way ANOVA, followed by a Tukey's multiple comparison test. Functional bioassay measurements, faecal pellet colonic motility and NO measurements were analysed using a two-way ANOVA followed by a Bonferroni's post-hoc test. Data was presented as mean \pm St.Dev. and $P<0.05$ was taken as significant.

\section{Results}

\subsection{Propofol alters CMMC function in the distal colon}

Representative traces of CMMC responses in the proximal and distal colon in response to $5 \mu \mathrm{M}$ propofol are shown in Figure 1A. No differences in the response of $1 \mu \mathrm{M}$ propofol was observed. There was a signficant reduction in the propagating velocity of CMMCs in $5 \mu \mathrm{M}$ propofol when compared to control (Figure 1B, $p<0.001$, $\mathrm{n}=6$ ). No additional reduction in the propagating velocity of CMMCs in $5 \mu \mathrm{M}$ propofol was observed when $100 \mu \mathrm{M}$ L-NNA was present.

In the proximal colon, there was no difference in the amplitude of CMMCs in the presence of $5 \mu \mathrm{M}$ propofol when compared to control (Figure $2 \mathrm{~A}, \mathrm{n}=6$ ). There was a signficant reduction in the CMMC amplitude in tissue perfused with $5 \mu \mathrm{M}$ propofol + $100 \mu \mathrm{M}$ L-NNA when compared to $5 \mu \mathrm{M}$ propofol alone $(\mathrm{p}<0.05, \mathrm{n}=6)$. No difference in the duration of CMMCs was observed in the proximal colon (Figure 2B).

In the distal colon, there was a signficant reduction in CMMC amplitude between control and $5 \mu \mathrm{M}$ propofol $(p<0.001, n=6$, Figure $2 \mathrm{C}$ ). No difference in the CMMC amplitude was observed when the tissue was exposed to $5 \mu \mathrm{M}$ propofol alone compared to $5 \mu \mathrm{M}$ propofol and $100 \mu \mathrm{M}$ L-NNA (Figure $2 \mathrm{C}$ ). None of the treatments altered the duration of CMMCs in the distal colon (Figure 2B). 


\subsection{Alterations in serotonin concentration response curves in proximal and distal colon following addition of propofol}

5-HT is a major driver of colonic motility and we were interested to examine if the effects of propofol could be explained by its effects on 5-HT evoked muscle contractions. Following addition of serotonin, a biphasic response was observed consisting of a fast relaxation followed by a sustained contraction.

Within the proximal colon there was no significant difference in the response in the presence of $5 \mu \mathrm{M}$ propofol when compared to control (Figure 3A). However, when studies were conducted in the presence of $5 \mu \mathrm{M}$ propofol $+100 \mu \mathrm{M}$ L-NNA, there was a significant increase in the integral of response $(p<0.01, n=6$, Figure $3 A)$. A signficant increase in the response in the presence of $100 \mu \mathrm{M}$ L-NNA was observed when responses were evoked with $10 \mu \mathrm{M}$ and $30 \mu \mathrm{M} 5-\mathrm{HT}(\mathrm{p}<0.01, \mathrm{n}=6)$.

Within the distal colon, there was a significant increase in the integral of the response observed in the presence of $5 \mu \mathrm{M}$ propofol when compared to control $(p<0.001, n=6$, Figure 3B). There was a significant increase in the integral of the response at 5-HT concentrations of $3 \mu \mathrm{M}(\mathrm{p}<0.05), 10 \mu \mathrm{M}(\mathrm{p}<0.01)$ and $30 \mu \mathrm{M}(\mathrm{p}<0.001, \mathrm{n}=6)$. No further increase was seen in the presence of $5 \mu \mathrm{M}$ propofol $+100 \mu \mathrm{M}$ L-NNA (Figure 3B).

\subsection{NO production in the distal colon was decreased in the presence of propofol}

NO production was evoked with a 10 second application of $10 \mu \mathrm{M}$ veratridine and a clear NO signal could be monitored on the BDD electrode (Figure 4A). No significant reduction in the concentration of NO production from myenteric neurons from proximal tissue was observed in the presence of $5 \mu \mathrm{M}$ propofol. NO production was, however, significantly reduced in the myenteric neurons from the distal colon in the presence of $5 \mu \mathrm{M}$ propofol $(\mathrm{p}<0.001, \mathrm{n}=4$, Figure 4B).

\subsection{Colonic motility is reduced by propofol}

Figure 5 shows the movement of the faecal pellet from the oral to anal end of the isolated colon comparing control and $5 \mu \mathrm{M}$ propofol. Under control conditions, the pellet migrated down the colon in step like movements, however in the presence of 5 
$\mu \mathrm{M}$ propofol, these movements ceased on reaching the distal colon. There was a significant reduction in the distance moved by the artificial faecal pellet in the presence of $5 \mu \mathrm{M}$ propofol (at 10 minutes $p<0.01$ and at 12,14 and 16 minutes $p<0.001, n=6$, Figure $5 \mathrm{~B}$ ). The was a significant reduction in the velocity of the artificial faecal pellet after the addition of $5 \mu \mathrm{M}$ propofol $(p<0.01, n=6$, Figure $1 C)$. 


\section{Discussion}

\subsection{Influence of propofol on colonic motility is through effects in the distal colon}

Our studies show that propofol at clinically relevant concentrations increased faecal pellet transit time and reduced colonic motility through reductions in NO production in the distal colon. Our findings clearly indicate that the effect of propofol has regionspecific effects in the colon as only reductions in CMMC amplitude and increase in 5HT-evoked contractions were observed in the presence of propofol only in the distal region of the colon. This is further strengthened by fecal pellet motility studies, where the artificial pellet moves through the proximal colon in a similar fashion as the control studies, but motility is halted at the mid point of the colon. Other published studies that have investigated the effect of propofol on gastrointestinal transit and human colonic muscle contraction have observed similar effects(Inada et al., 2004; Lee et al., 1999), but were unable to provide any indication as to whether these effects were region specific.

\subsection{Effects of nitrergic signalling processes}

As shown in Figure 2, propofol only decreases CMMC amplitude in the distal colon and overall reduced the velocity of CMMCs in the colon. Previous studies that have explored the effect of L-NNA on the murine colon, have shown that inhibition of NO production following application of nitric oxide synthase blockers NOLA (Spencer, 2001) and L-NNA (Fida et al., 1997; Powell et al., 2001) results in a reduction in CMMC amplitude and velocity. This suggests that propofol is inhibiting nitrergic signalling. These effects were not observed in the proximal colon as the amplitude was not altered in the presence of propofol but was reduced when L-NNA was added, further supporting that the effects of propofol in the distal colon are through an inhibition of nitrergic signalling.

Similar responses were observed when studies were conducted to explore the changes in 5-HT-evoked contractions. $5-\mathrm{HT}$ is known to have biphasic effects on the colon, causing an initial relaxation followed by a sustained contraction. No alteration in the 5-HT response was observed in the presence of propofol in the proximal colon, but this response was significantly increased when L-NNA was added, suggestive of 
a functional nitrergic pathway which was not altered by propofol. In the distal colon, however, the 5-HT evoked response was significantly increased in the presence of 5 $\mu \mathrm{M}$ propofol and could not be further increased through the addition of L-NNA. Together these data indicate that propofol appears to have its effects through a suppression of nitrergic signalling in the distal colon. These functional changes are most likely due to alterations in NO production, which may occur through inhibiting nitric oxide synthase (NOS), guanylyl cyclase (GC) receptor or ion channels.

Based on the observation that the veratridine-evoked NO signal is reduced by propofol in the distal colon, it is most likely that propofol inhibits NOS to reduce NO production, leading to an increased contractile state of the colon, which increases transit time. Previous studies in various biological regions other than the gastrointestinal tract have contradictory findings with some studies indicating that propofol either inhibits the expression and activity of NOS(Chen et al., 2003; Lv et al., 2013), whilst many others indicate that propofol induces relaxation by stimulating $\mathrm{NO}$ production in the endothelium(Park et al., 1992; Toda et al., 2007).

Our results show that propofol has an effect in the distal colon and not in the proximal colon. This indicates that propofol may induce region-specific differences on motility. Nitrergic neuronal function is diverse in different regions of the colon(Toda et al., 2005). Studies on CMMC function show L-NNA increased CMMC amplitude in the mid and distal regions of the colon (Brierley et al., 2001; Powell et al., 2001; Powell et al., 2002). However, there was either no effect or decreased amplitude in the proximal region. These results suggest NOS signalling mechanisms along the colon are complex and that the proximal colon is more resilient to propofol than the distal colon.

The central actions of propofol through its ability to bind to and stimulate $\mathrm{GABA}_{A}$ receptor inhibitory tone (Trapani et al., 2000). It is therefore possible that the ability of propofol to reduce relaxation in the $\mathrm{Gl}$ tract are due to its well-known actions on $\mathrm{GABA}_{A}$ receptors. However, in the $\mathrm{GI}$ tract stimulation of $\mathrm{GABA}_{A}$ receptors excites myenteric neurons rather than inhibiting them (Erdö, 1985). Previous studies have demonstrated the presence of GABAA receptors on many enteric neurons and enteroendocrine cells including NOS positive enteric neurons (Jessen et al., 1979). Therefore, if propofol's actions on the distal colon were through its ability to potentiate the GABAA receptor 
stimulation we would predict an increased in NO-mediated relaxation in the GI tract rather than the reduction observed in this study.

Additionally, propofol has been shown to inhibit 5- $\mathrm{HT}_{3}$ receptor signalling (Barann et al., 2008). 5- $\mathrm{HT}_{3}$ receptors are present on inhibitory nitrergic neurons and are the target for descending inhibitory serotonergic interneurons (Dickson et al., 2010; Heredia et al., 2013). A reduction in 5- $\mathrm{HT}_{3}$ receptor signalling by propofol would therefore reduce nitrergic signalling consistent with our study findings and explain the reduction in distal colon motility.

\subsection{Clinical implication of the role of propofol on colonic motility}

The results of our study showed that at clinically relevant doses, propofol reduces colonic motility in C57BL/6 mice through an inhibition of nitrergic signalling in the distal colon. Such changes would be indicative of constipation symptoms. Propofol has not been reported in the literature to cause constipation as it is often difficult to find a contribution of propofol to aetiology when there are so many possible contributors such as the type of anaesthesia used, surgery conducted or other factors such as postoperative ileus(Kehlet et al., 2001).

There have been very few clinical studies investigating the effects of propofol on gastrointestinal transit in hospital patients and those few have only looked at the postoperative effects of propofol. Although these studies propose that propofol has only a very minor effect on motility, diagnosis of motility disturbances can be challenging in the critically ill when they are on multiple medications. Propofol infusion in critically ill patients who were fed enterically caused an increase in gastric residual volume however gastric transit remained unchanged(Memiş et al., 2006).

Understanding the mechanisms by which propofol decreases colonic motility may be helpful in providing treatments for possible side effects of reduced motility from propofol use. Finding out how long the effect on motility lasts and whether it is dosedependent with higher doses than used in this study may determine how propofol is prescribed and administered for maintenance and sedation in theatre, intensive care units (ICU) and the palliative care setting.

\subsection{Conclusion}


At clinically relevant doses, propofol was shown to reduce faecal pellet motility by altering $\mathrm{CMMC}$ and $5-\mathrm{HT}$-induced contraction, as well as reducing $\mathrm{NO}$ production in the distal colon. Our findings indicate that propofol inhibits NOS, therefore reducing NO production, which leads to dysmotility. Overall our studies indicate that propofol has a significant influence on distal colon signalling mechanisms and impairs colonic motility, which may have implications in its clinical use. Further studies may help to determine its significance in contributing to prolonged post-op ileus in hospital patients and help determine treatments.

\section{Acknowledgements}

The authors would like to thank the team in the bioresources unit.

Author contribution - Lucy B Diss: This author conducted some experimental studies, was responsible for analysis of the experimental data and writing of the manuscript. Shannon Villeneuve: This author conducted and analysed functional bioassay data. Kim R Pearce: This author conducted and analysed functional bioassay data. Mark S Yeoman: This author assisted on experimental design, data analysis and critical review of the manuscript. Bhavik A Patel: This author conceived the study and assisted on experimental design and analysis, as well as writing of the manuscript. All authors approved the final version of the manuscript.

Financial Disclosures: None

Conflicts of interest: None

\section{References}


Barann, M., Linden, I., Witten, S., Urban, B.W. 2008. Molecular actions of propofol on human 5-HT3A receptors: enhancement as well as inhibition by closely related phenol derivatives. Anesthesia \& Analgesia 106, 846-857.

Beller, J.P., Pottecher, T., Lugnier, A., Mangin, P., Otteni, J.C. 1988. Prolonged Sedation With Propofol In Icu Patients: Recovery And Blood Concentration Changes During Periodic Interruptions In Infusion. British Journal of Anaesthesia 61, 583-588. Brierley, S.M., Nichols, K., Grasby, D.J., Waterman, S.A. 2001. Neural mechanisms underlying migrating motor complex formation in mouse isolated colon. $\mathrm{Br} \mathrm{J}$ Pharmacol 132, 507-517.

Chen, R.M., Wu, G.J., Tai, Y.T., Sun, W.Z., Lin, Y.L., Jean, W.C., Chen, T.L. 2003. Propofol reduces nitric oxide biosynthesis in lipopolysaccharide-activated macrophages by downregulating the expression of inducible nitric oxide synthase. Arch Toxicol 77, 418-423.

Dickson, E.J., Heredia, D.J., Smith, T.K. 2010. Critical role of 5-HT1A, 5-HT3, and 5HT7 receptor subtypes in the initiation, generation, and propagation of the murine colonic migrating motor complex. Am J Physiol Gastrointest Liver Physiol 299, G144157.

Erdö, S.L. 1985. Peripheral GABAergic mechanisms. Trends in Pharmacological Sciences 6, 205-208.

Favetta, P., Degoute, C.S., Perdrix, J.P., Dufresne, C., Boulieu, R., Guitton, J. 2002. Propofol metabolites in man following propofol induction and maintenance. BJA: British Journal of Anaesthesia 88, 653-658.

Fida, R., Lyster, D.J., Bywater, R.A., Taylor, G.S. 1997. Colonic migrating motor complexes (CMMCs) in the isolated mouse colon. Neurogastroenterol Motil 9, 99-107. 
Freye, E., Sundermann, S., Wilder-Smith, O.H. 1998. No inhibition of gastro-intestinal propulsion after propofol- or propofol/ketamine-N2O/O2 anaesthesia. A comparison of gastro-caecal transit after isoflurane anaesthesia. Acta Anaesthesiol Scand 42, 664669.

Heredia, D.J., Gershon, M.D., Koh, S.D., Corrigan, R.D., Okamoto, T., Smith, T.K. 2013. Important role of mucosal serotonin in colonic propulsion and peristaltic reflexes: in vitro analyses in mice lacking tryptophan hydroxylase 1. The Journal of physiology $591,5939-5957$.

Inada, T., Asai, T., Yamada, M., Shingu, K. 2004. Propofol and Midazolam Inhibit Gastric Emptying and Gastrointestinal Transit in Mice. Anesthesia \& Analgesia 99, $1102-1106$.

Jessen, K.R., Mirsky, R., Dennison, M.E., Burnstock, G. 1979. GABA may be a neurotransmitter in the vertebrate peripheral nervous system. Nature 281, 71.

Kehlet, H., Holte, K. 2001. Review of Postoperative Ileus. The American Journal of Surgery 182, S3-S10.

Lee, T.L., Ang, S.B., Dambisya, Y.M., Adaikan, G.P., Lau, L.C. 1999. The effect of propofol on human gastric and colonic muscle contractions. Anesth Analg 89, 12461249

Lv, F., Shen, Y.-w., Peng, L.-h., Li, P., Luo, J., Wei, K., Li, W., Chen, J., Min, S. 2013. Effects of Propofol on Expression of Hippocampal Neuronal Nitric Oxide Synthase and Carboxy-Terminal PDZ Ligand of Neuronal Nitric Oxide Synthase in Stressed Rats Undergoing Electroconvulsive Shock. The Journal of ECT 29, 297-302.

MacEachern, S.J., Patel, B.A., Keenan, C.M., Dicay, M., Chapman, K., McCafferty, D.-M., Savidge, T.C., Beck, P.L., MacNaughton, W.K., Sharkey, K.A. 2015. Inhibiting 
Inducible Nitric Oxide Synthase in Enteric Glia Restores Electrogenic Ion Transport in Mice With Colitis. Gastroenterology 149, 445-455.e443.

Memiş, D., Dökmeci, D., Karamanlioğlu, B., Turan, A., Türe, M. 2006. A comparison of the effect on gastric emptying of propofol or dexmedetomidine in critically ill patients: preliminary study. Eur J Anaesthesiol 23, 700-704.

Park, M.D.Wyun K., Lynch, M.D.P.D.C., Johns, M.D.Roger A. 1992. Effects of Propofol and Thiopental in Isolated Rat Aorta and Pulmonary Artery. Anesthesiology 77, 956-963.

Patel, B.A. 2011. Electroanalytical approaches to study signaling mechanisms in the gastrointestinal tract. Neurogastroenterology \& Motility 23, 595-605.

Patel, B.A., Arundell, M., Parker, K.H., Yeoman, M.S., O'Hare, D. 2006. Detection of Nitric Oxide Release from Single Neurons in the Pond Snail, Lymnaea stagnalis. Analytical Chemistry 78, 7643-7648.

Patel, B.A., Galligan, J.J., Swain, G., Bian, X. 2008. Electrochemical monitoring of nitric oxide released by myenteric neurons of the guinea pig ileum. Neurogastroenterology \& Motility 20, 1243-1250.

Powell, A.K., Bywater, R.A. 2001. Endogenous nitric oxide release modulates the direction and frequency of colonic migrating motor complexes in the isolated mouse colon. Neurogastroenterol Motil 13, 221-228.

Powell, A.K., O'brien, S.D., Fida, R., Bywater, R.A. 2002. Neural integrity is essential for the propagation of colonic migrating motor complexes in the mouse. Neurogastroenterol Motil 14, 495-504.

Rowe, K., Fletcher, S. 2008. Sedation in the intensive care unit. Continuing Education in Anaesthesia Critical Care \& Pain 8, 50-55. 
Russell, G.N., Wright, E.L., Fox, M.A., Douglas, E.J., Cockshott, I.D. 1989. Propofolfentanyl anaesthesia for coronary artery surgery and cardiopulmonary bypass. Anaesthesia 44, 205-208.

Schnoor, J., Unger, J.K., Kuepper, T., Bode, B., Hofeditz, A., Silny, J., Rossaint, R. 2005. Effects of propofol and fentanyl on duodenal motility activity in pigs. The Canadian veterinary journal $=$ La revue veterinaire canadienne 46, 995-1001.

Spencer, N.J. 2001. Control of migrating motor activity in the colon. Curr Opin Pharmacol 1, 604-610.

Toda, M.D.P.D.N., Toda, M.D.P.D.H., Hatano, M.D.P.D.Y. 2007. Nitric Oxidelnvolvement in the Effects of Anesthetic Agents. Anesthesiology 107, 822-842. Toda, N., Herman, A.G. 2005. Gastrointestinal function regulation by nitrergic efferent nerves. Pharmacol Rev 57, 315-338.

Trapani, G., Altomare, C., Sanna, E., Biggio, G., Liso, G. 2000. Propofol in anesthesia. Mechanism of action, structure-activity relationships, and drug delivery. Current medicinal chemistry $7,249-271$.

Venn, R.M., Grounds, R.M. 2001. Comparison between dexmedetomidine and propofol for sedation in the intensive care unit: patient and clinician perceptions $†$. BJA: British Journal of Anaesthesia 87, 684-690. 


\section{Figure Legends}

Figure 1. Recording of CMMCs. (A) Representative traces of CMMC recording in the proximal $(P)$ and distal $(D)$ region of the isolated colon. The responses to $5 \mu \mathrm{M}$ propofol and $5 \mu \mathrm{M}$ propofol + L-NNA are shown. $(B)$ the propagating velocity of a CMMC is shown in the presence of $5 \mu \mathrm{M}$ propofol and $100 \mu \mathrm{M}$ L-NNA. Data shown as mean \pm St.Dev., where ${ }^{* *} p<0.001$.

Figure 2. Responses of CMMC amplitude and duration in the presence of $5 \mu \mathrm{M}$ propofol. Response of the CMMC amplitude $(A)$ and duration $(B)$ from the proximal colon. Response of the CMMC amplitude (C) and duration (D) from the distal colon. Data shown as mean \pm St.Dev., ${ }^{*} p<0.05$ and ${ }^{* * *} p<0.001$.

Figure 3. 5-HT-evoked concentration response curves. (A) proximal and (B) distal colon responses to $5-\mathrm{HT}$ evoked contraction in the presence and absence of $5 \mu \mathrm{M}$ propofol and $5 \mu \mathrm{M}$ propofol $+100 \mu \mathrm{M}$ L-NNA. Data shown as mean \pm St.Dev., $\mathrm{n}=6$, ${ }^{*} p<0.05,{ }^{* *} p<0.01$ and ${ }^{* * *} p<0.001$ vs control; and ${ }^{\ddagger \ddagger} p<0.01$ vs $5 \mu$ M propofol.

Figure 4. NO production in the presence of propofol. (A) shows responses of NO production evoked using $10 \mu \mathrm{M}$ veratridine and (B) shows response of NO observed in proximal and distal colon in the presence of propofol. Grey bar indicates the duration of action of veratridine. Data shown as mean \pm St.Dev., $n=4,{ }^{* * *} p<0.001$

Figure 5. Colonic pellet motility study (A) shows the movement of the faecal pellet tracked through video imaging. $(B)$ the distance that the pellet moved down the colon was measured every 2 minutes in the presence and absence of $5 \mu \mathrm{M}$ propofol. (C) The velocity of the pellet in the presence and absence of $5 \mu \mathrm{M}$ propofol. Data shown as mean \pm St.Dev., $n=6$, where ${ }^{* *} p<0.01$ and ${ }^{* * *} p<0.001$. 
Figures

Figure 1

A
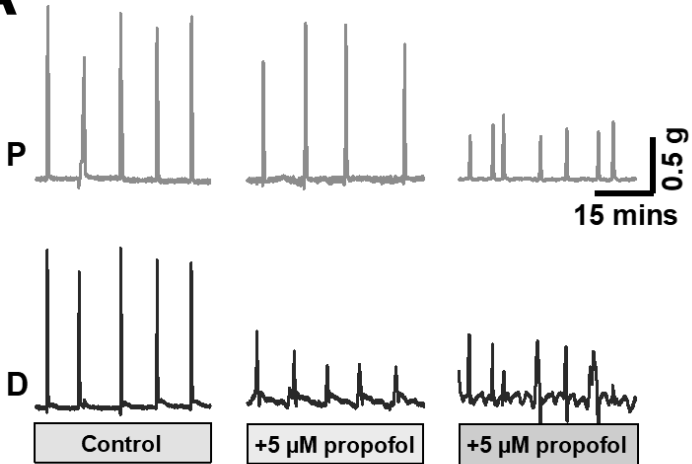

$+5 \mu \mathrm{M}$ propofol
$\substack{+5 \mu \mathrm{M} \text { propofol } \\+100 \mu \mathrm{M} \text { L-NNA }}$

B

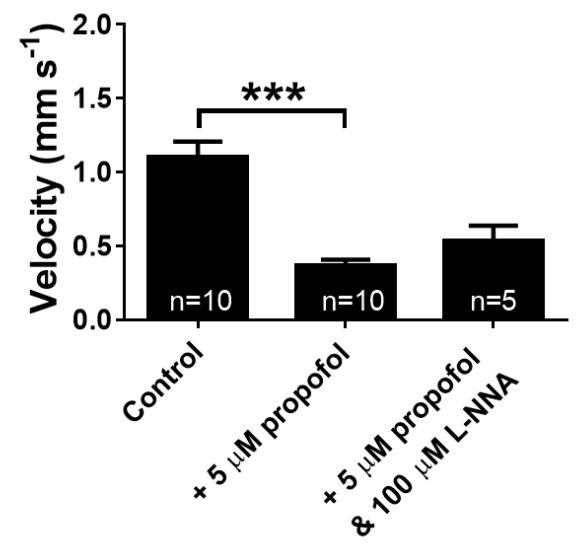


Figure 2

\section{Proximal Colon}

A

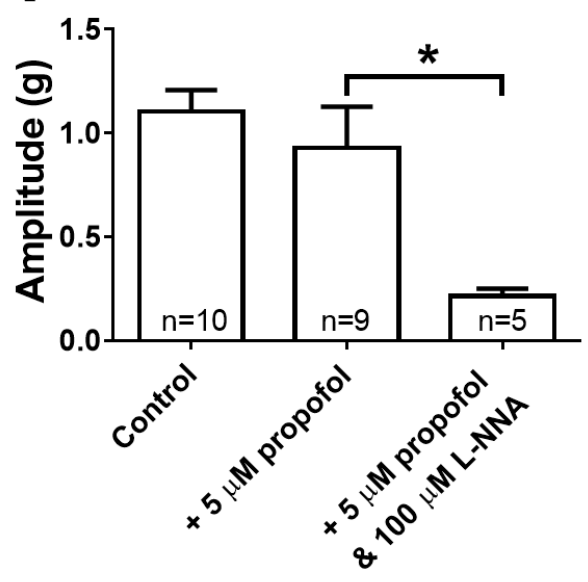

$B$

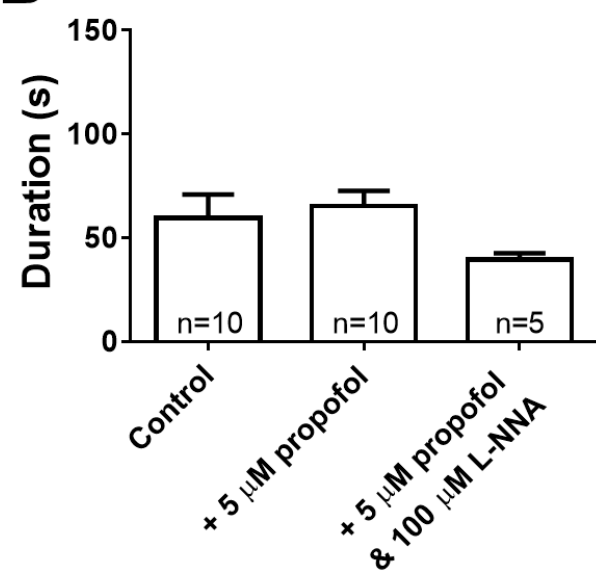

Distal Colon

C

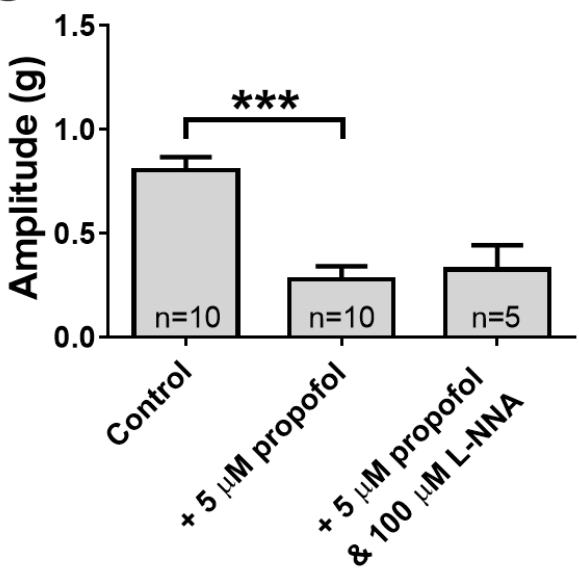

D

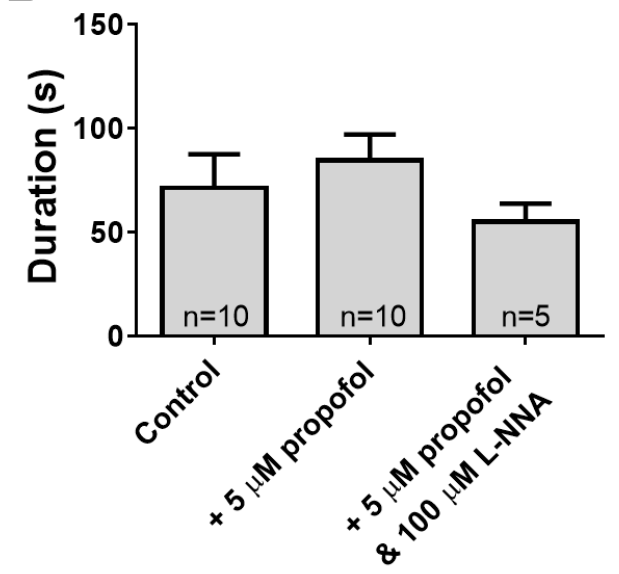


Figure 3

\section{A Proximal Colon}
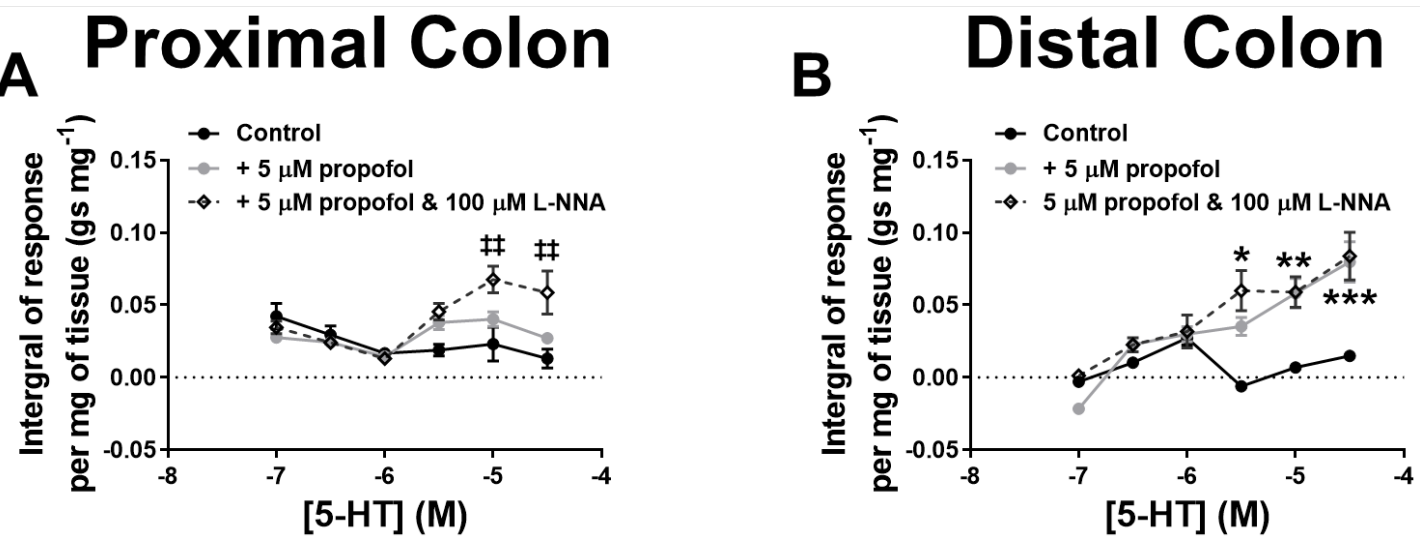
Figure 4
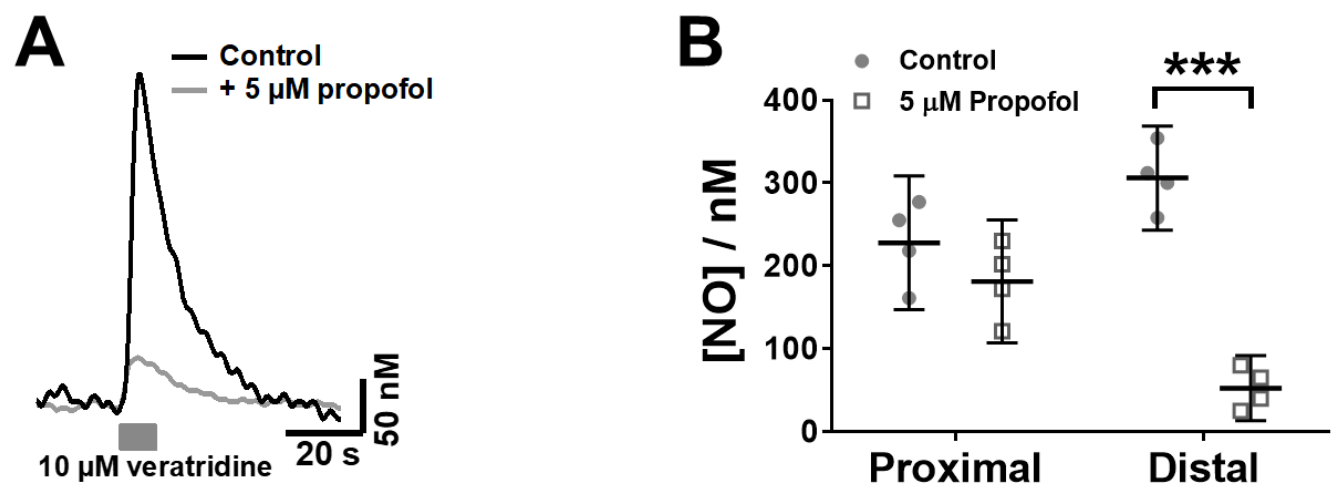
Figure 5

A

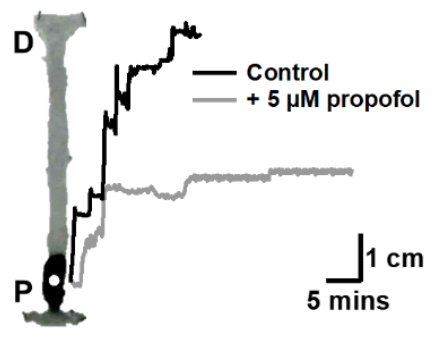

B

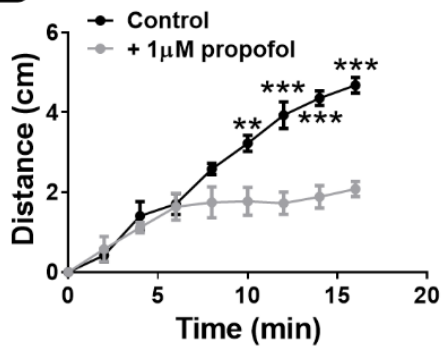

C

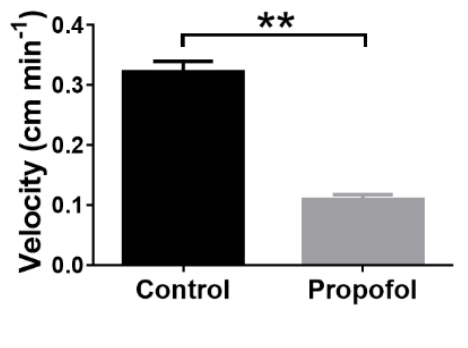

